\title{
COMPARAÇÃO DA TAXA DE DEPOSIÇÃO DO ARAME SÓLIDO ER70S6, 1,2MM, NO PROCESSO GMAW (MAG), COM O ARAME TUBULAR E71T1, 1,2MM, NO PROCESSO FCAW NO AÇO ASTM A 36
}

\author{
COMPARISON of DEPOSITION RATE ERTOS6 SOLID WIRE, 1, $2 \mathrm{~mm}$ in GMAW \\ (MAG), with the E71T1, CORED WIRE 1, 2 mm FCAW process in STEEL ASTM A 36
}

\author{
Merciane Miranda Vulcão - vulcaomerciane@gmail.com \\ Marco Aurélio de Carvalho Silva - marcosilva192@gmail.com \\ Estudantes do Curso Superior de Tecnologia em Mecânica: processos de soldagem \\ Faculdade de Tecnologia (FATEC) - Sertãozinho - São Paulo - Brasil \\ Prof. Me. Edmilson Antonio Sarni - edmilsonsarni@hotmail.com \\ Profa. Pós-Dra. Maria Aparecida Bovério - mariaboverio@ hotmail,com \\ Faculdade de Tecnologia (FATEC) - Sertãozinho - São Paulo - Brasil
}

\section{RESUMO}

O presente artigo apresenta uma análise comparativa da taxa de deposição do arame sólido AWS A5.18 ER70S-6 e tubular AWS A5.20 E71T-1 respectivamente nos processos de soldagem Gás Metal Arc Welding (GMAW) na variante Metal Active Gás (MAG) e Flux Cored Arc Welding $(F C A W)$. Atualmente ambos os arames são utilizados para soldagem a arco em juntas de aços comuns com baixo e médio teor de carbono, porém com um crescimento substancial no uso do tubular em relação ao sólido. O objetivo desta pesquisa foi comparar o processo de soldagem $G M A W$ e $F C A W$ com os arames maciço e tubular, verificando as três variáveis que podem ser analisadas como indicativos de produtividade em soldagem: fator de operação, taxa de deposição e tempo de soldagem. Neste estudo as soldas foram feitas na posição plana em chanfro em V, no aço carbono ASTM A-36 para o delineamento dos experimentos e, em seguida, análise da influência dos parâmetros: tensão, corrente e tempo de soldagem em ambos os processos. Como procedimentos metodológicos foram utilizadas a pesquisa bibliográfica e experimental, por meio de laboratório. Os resultados permitem concluir que a taxa de deposição foi superior para o arame ER70S-6 em relação ao arame E71T-1, em função da corrente elétrica, onde, é a fundamental variável para o aumento da taxa de deposição. Outra variável que se destacou na menor redução de tempo de soldagem, gerando, portanto, uma maior produtividade do processo FCAW em relação ao GMAW.

Palavras-chave: Soldagem. Taxa de deposição. GMAW. FCAW. Metal de Adição e MAG.

\begin{abstract}
This article presents a comparative analysis of deposition rate of solid wire AWS ER70S-6 's 5.18 and 5.20 E71T-tubular AWS to 1 respectively in the Metal Gas welding processes Arc
\end{abstract}




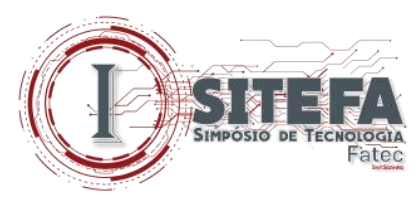

Welding (GMAW) on Metal variant Active gas (MAG) and Flux Cored Arc Welding ( FCAW). Currently both wires are used for arc welding in common steel joints with low and medium carbon content, but with a substantial growth in the use of tubular compared to solid. The objective of this research was to compare the GMAW and FCAW welding process with solid and tubular wires, checking the three variables that can be analyzed as indicators of productivity in soldering: operating factor, deposition rate and welding time. In this study the welds were made in flat position in V-Groove, carbon steel ASTM A-36 to the outline of the experiments and then analyze the influence of the parameters: voltage, current and welding time in both processes. As methodological procedures were used to bibliographical research and experimental, through lab. The results allow to conclude that the rate of deposition was superior to the wire ER70S-6 wire E71T-compared to 1, depending on the electric current, where, is the fundamental variable for the increased rate of deposition. Another variable that stood out in the lower welding time reduction, generating thus higher productivity of FCAW process compared to GMAW.

Keywords: Welding. Deposition rate. GMAW. FCAW. Filler metals and MAG.

\section{DOI:}

\section{INTRODUÇÃO}

$\mathrm{Na}$ indústria Metalmecânica uma das principais metas é a busca por maior produtividade com menor custo de fabricação. Nessa perspectiva, o aumento da participação dos processos de soldagem com arame tubular e arame sólido em todo mundo corresponde a essas metas e isso vem ocorrendo porque os processos de soldagem a arco elétrico com atmosfera protetora têm apresentado um contínuo desenvolvimento devido a sua qualidade, produtividade, flexibilidade e melhor adequação à mecanização.

Nesse sentido este artigo apresenta a pesquisa comparativa com os processos de soldagem Gás Metal Arc Welding (GMAW) na variante Metal Active Gás (MAG) e Flux Cored Arc Welding (FCAW), os quais tornaram-se amplamente utilizados na indústria metalúrgica. Esses dois processos podem ser utilizados em diversos tipos de aços: aços de baixo e médio carbono, os aços inoxidáveis entre diversas ligas ferrosas. Um ponto significativo desses dois processos é sua surpreendente produtividade quando há a automação dos equipamentos, sendo que na indústria eles são amplamente empregados desta maneira.

Para a correta compreensão sobre a escolha do processo que vai gerar a produtividade desejada para esta pesquisa, optou-se por comparar os processos de soldagem $G M A W$ na variante $M A G$ e $F C A W$. Utilizou-se o processo $G M A W$ com arame sólido AWS ER 70S6 e o 


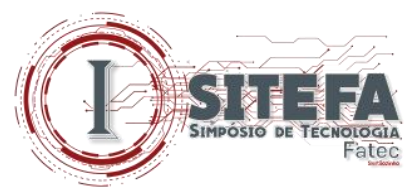

FCAW com o arame tubular AWS E71T-1. Ambos são significativamente utilizados por sua excelente produtividade no ramo da produção metalúrgica, devido às suas equivalências, nível de desempenho e tipos de equipamentos utilizados. Os dois são processos de soldagem por fusão com arame alimentado de forma contínua e arco elétrico sendo estabelecido entre o consumível e a peça.

O processo de soldagem GMAW com arame sólido AWS ER 70S6 é empregado exclusivamente na soldagem de matérias ferrosos, compondo-se de gás de proteção com a mistura $75 \%$ AR $25 \% \mathrm{CO}_{2}$. O processo de soldagem FCAW com arrame tubular AWS E $71 \mathrm{~T} 1$ também é utilizado na soldagem de materiais não ferrosos e seu gás de proteção pode ser $100 \% \mathrm{CO}_{2}$ ou misturas. Os dois são bastante equivalentes em seus princípios de desempenho e nos tipos de equipamentos utilizados.

A literatura pertinente a esta temática relaciona alguns aspectos operacionais formando comparações entre os processos de soldagem por taxa de deposição na posição plana, onde nem sempre coincide com a capacidade operacional dos processos. Nesse sentido, considerouse oportuna essa pesquisa, a fim de investigar uma metodologia que seja possível analisar de maneira comparativa os dois processos, para uma energética aplicação e condição de soldagem.

O objetivo desta pesquisa é comparar o processo de soldagem GMAW na variante $M A G$ e $F C A W$ com os arames sólido e tubular, verificando as três variáveis que podem ser analisadas como indicativos de produtividade em soldagem: fator de operação, taxa de deposição e tempo de soldagem.

$\mathrm{Na}$ organização deste artigo é apresentada na seção 2 a revisão bibliográfica concernente à tecnologia da soldagem, a seção 3 apresenta os procedimentos metodológicos da pesquisa: materiais e métodos, a seção 4 dedica-se aos resultados e discussões e a seção 5 apresenta a conclusão da pesquisa.

\section{TECNOLOGIA DA SOLDAGEM}

A história da tecnologia de soldagem apresenta uma permanente vocação para o uso pioneiro de descobertas e inovações vindas dos laboratórios científicos, mostrando com frequência sua inquietude ante novas descobertas, num processo cada vez mais acelerado na 


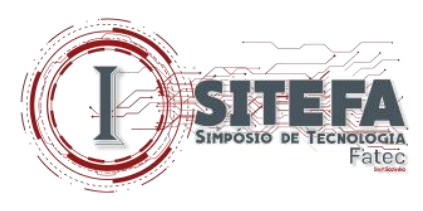

procura da sua aplicação prática. As expressões como eficiência, produtividade, tecnologia são de uso corrente na indústria pelos técnicos. (WAINER; BRANDI; MELLO, 2015).

Bracarense, Modenesi e Marques (2009) explicam que como um importante processo a soldagem utilizada para união de metais na indústria vai desde sua aplicação em microeletrônicos a fabricação de equipamentos de centenas ou milhares de toneladas de peso. De acordo com Wainer, Brandi e Mello (2004) a soldagem é predominante para o processo da união entre dois metais utilizando-se da fonte de calor e aplicando-se ou não pressão.

Os processos de soldagem são classificados conforme as normas da Associação Brasileira de Normas Técnicas (ASSOCIAÇÃO BRASILEIRA DE NORMAS TÉCNICAS) e através de uma padronização de números e nomes de processos de soldagem criou-se uma norma técnica, a NBR:13043. (ABNT, 1993).

O aço ASTM A36 é a explanação mais clássica de um aço estrutural de grãos finos. É um aço carbono ferrítico, ligado ao manganês, com teores de fósforos e enxofre controlados, alta resistência e baixa liga, empregados em partes estruturais nos quais a economia em peso é notável. (COLPAERT, 2008).

A soldagem com arame tubular contém inúmeros traços em relação ao processo GMAW no que corresponde aos equipamentos e princípios de funcionamento. Esta situação lhe permite partilhar o fator de trabalho e a taxa de deposição característicos da soldagem $G M A W$. No entanto, através da soldagem $F C A W$ é capaz de obter a alta versatilidade da soldagem com eletrodo revestido na regulagem da composição química, facilidade de trabalho em campo. (GOMES, 2006).

Os arames tubular e sólido contêm grande semelhança quanto aos equipamentos utilizados na soldagem bem como de aplicações recomendadas. Uma das particularidades como alta produtividade, boa flexibilidade e facilidade de operação reintegram os processos GMAW e $F C A W$ fortemente competitivos e, em muitas circunstâncias, são concorrentes diretos nas aplicações de soldagem. (RODRIGUES; PAIVA; COSTA, 2008).

Luttler e Stapon (1999 apud GOMES, 2006) explicam que o gás de proteção interage com arame tubular na soldagem melhorando a resistência mecânica, a dureza e a resistência a corrosão no metal depositado. Se for administrada a transferência hábil dos componentes do arame tubular, tanto a microestrutura como propriedades inerentes as soldagens podem ser 


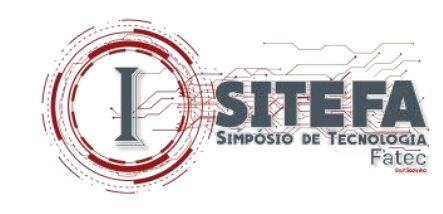

transmudadas pelo gás de proteção, além de afetar o conteúdo residual de hidrogênio e oxigênio e nitrogênio dissolvido no metal de solda.

Gomes (2006) afirma que tanto a literatura como as observações práticas permitem inferir que a eficiência é a compatibilidade entre a taxa de deposição e o consumo. A aplicação e a quantidade de arame fundido por unidade de tempo, por sua vez, diz respeito a taxa de deposição e a quantidade de arame (em peso) depositado por unidade de tempo. A taxa de deposição necessita de algumas variáveis de soldagem, tais como: diâmetro do arame, composição, e extensão do arame de soldagem.

\section{PROCEDIMENTOS METODOLÓGICOS}

Os procedimentos metodológicos adotados nesta pesquisa fundamentam-se na pesquisa bibliográfica que explorou e obteve os conhecimentos necessários inerentes às tecnologias de soldagem, e assim como definem Barros e Lehfeld (1986) procurou-se encontrar as informações já publicadas na literatura existente.

A partir do conhecimento sobre as tecnologias de soldagem realizou-se a pesquisa laboratorial, entendida conforme definição de Prodanov e Freitas (2013) como aquela que é realizada em situações controladas, em laboratórios, pois precisa de um ambiente de acordo com o estudo a ser desenvolvido, uma vez que o contexto é controlado pelo ambiente de laboratório.

\subsection{Materiais e métodos}

Para realização desta pesquisa foram utilizados dois tipos de arames, para o processo de soldagem GMAW arame sólido cobreado (AWS A5.18 ER70S-6) com diâmetro de 1,2 mm e para o processo de soldagem $F C A W$ o arame tubular (AWS A5.20 E71T-1) com diâmetro de 1,2 mm. Suas composições químicas são especificadas na tabela 1 conforme certificado, os quais foram utilizados no experimento. 


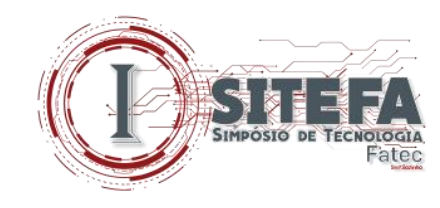

Tabela 1 - Composição química dos arames maciço ER70S-6 e arame tubular E71T-1

\begin{tabular}{cccccccccc}
\hline Material & $\mathbf{C \%}$ & $\mathbf{S i} \%$ & $\mathbf{M n \%}$ & $\mathbf{P \%}$ & $\mathbf{S \%}$ & $\mathbf{C u \%}$ & $\mathbf{N i \%}$ & $\mathbf{C r} \%$ & $\mathbf{M o \%}$ \\
\hline ER70S-6 & 0,086 & 0,86 & 1,57 & 0,014 & 0,012 & 0,018 & 0,019 & 0,016 & 0,006 \\
Especificações & $0,06-0,15$ & $0,80-1,15$ & $0,04-0,85$ & $\leq 0,025$ & $\leq 0,035$ & $\leq 0,5$ & $\leq 0,15$ & $\leq 0,15$ & $\leq 0,15$ \\
AWS & 0,04 & 0,45 & 1,30 & 0,01 & 0,01 & & & & \\
E71T-1 & $\leq 0,12$ & $\leq 0,9$ & $\leq 1,75$ & $\leq 0,03$ & $\leq 0,03$ & $\leq 0,35$ & $\leq 0,5$ & $\leq 0,2$ & $\leq 0,3$ \\
Especificações & & & & & & & & \\
AWS & & & &
\end{tabular}

Fonte: Lincoln Electric Brasil (2016)

Os corpos de prova foram confeccionados em chapa de aço ASTM A36 para realização do estudo, as dimensões do metal de base foram: com espessura de 1/2" $(12,70 \mathrm{~mm})$, $100,0 \mathrm{~mm}$ de largura e $250 \mathrm{~mm}$ de comprimento e carbono equivalente 0,298 (AWS). A tabela 2 apresenta a análise química do certificado do aço utilizado no experimento.

Tabela 2 - composição química do aço ASTM-A36

\begin{tabular}{llllllllll}
\hline $\mathrm{C}$ & $\mathrm{Mn}$ & $\mathrm{Si}$ & $\mathrm{P}$ & $\mathrm{S}$ & $\mathrm{Al}$ & $\mathrm{Cr}$ & $\mathrm{Ti}$ & $\mathrm{Ni}$ & $\mathrm{V}$ \\
0,15 & 0,67 & 0,20 & 0,013 & 0,008 & 0,019 & 0,01 & 0,002 & 0,01 & 0,002 \\
\hline \multicolumn{10}{c}{ Fonte: Gerdau (2017) }
\end{tabular}

Para realização do estudo, as amostras utilizadas foram pesadas antes e depois da soldagem, em uma balança de precisão, no preparo das amostras teve-se o cuidado procurando isentá-la de óleo, graxa, ferrugem, considerando uma faixa de $25 \mathrm{~mm}$ de cada lado da área do cordão de solda. Para realização do processo de soldagem $G M A W$ foi utilizado uma mistura de gás de proteção ativo contendo $75 \%$ de argônio e $25 \%$ de $\mathrm{CO}_{2}$ como vazão de 12 à 15 litros/min, executado com um equipamento de soldagem convencional $M A G$ da marca LINCON ELECTRIC modelo Power Wave S 350 com display digital, com tensão de soldagem de 29,0 V e corrente de soldagem de 200 A, pois nessas condições, o processo apresenta um arco mais estável e uma maior penetração. $\mathrm{O}$ alimentador do arame foi feito com cabeçote externo modelo LF 45, também da marca LINCON ELECTRIC. Durante a execução da soldagem a velocidade de alimentação de arame 6,8 metros por minuto. Já no processo $F C A W$ o equipamento de soldagem foi o mesmo mudando apenas os seguintes parâmetros de soldagem: a proteção gasosa feita com $100 \%$ de $\mathrm{CO}_{2}$ com vazão de 18 a 25 litros/min, a tensão de soldagem de $25 \mathrm{~V}$, a corrente de $200 \mathrm{~A}$, a velocidade de alimentação do arame de 8,5 metros por minuto. 


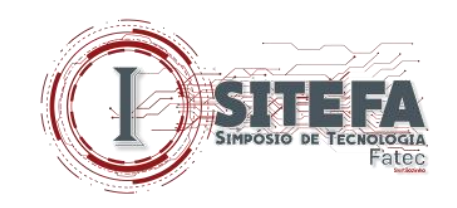

Os corpos de provas estudados foram de aço ASTM A36 com formação de um ângulo total do chanfro de $60^{\circ}$. Para preenchimento total da junta a ser soldada foram feitos cinco passes, com a seguinte ordem de execução: raiz, enchimento e acabamento de um lado; goivagem e acabamento do lado oposto, conforme fotografias 1a, 1b, 1c, 1d e 1e. Para o processo GMAW com arame ER70S-6 e nas fotografias 2a, 2b, 2c, 2d e 2e para o processo FCAW com arame E71T-1.

Fotografias 1a, 1b, 1c, 1d e 1e - Processo de soldagem GMAW com arame ER70S-6
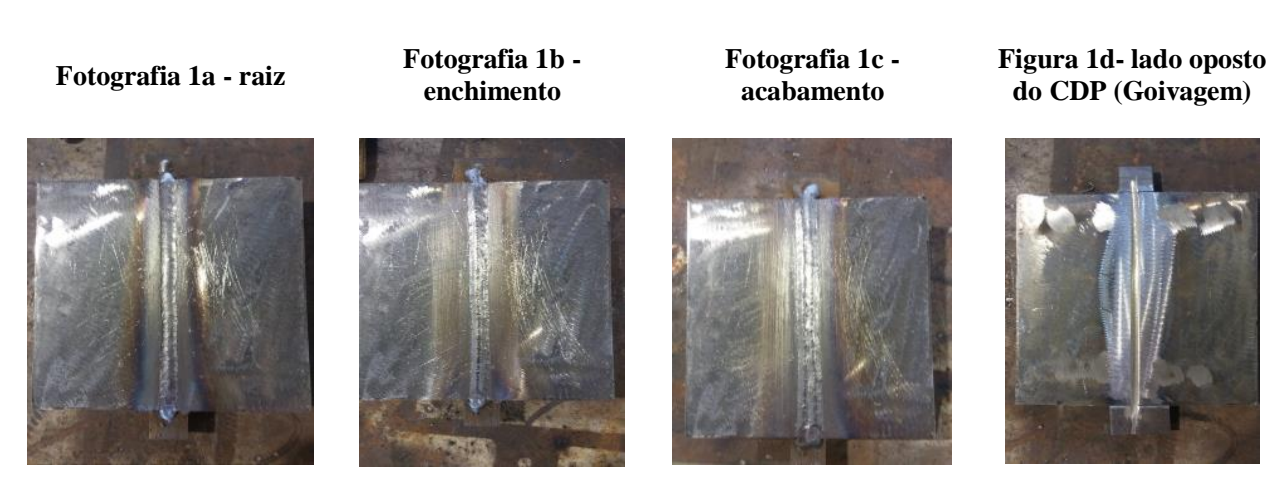

Figura 1e acabamento do lado oposto do CDP oposto do CDP
(Goivagem)

Fonte: Os Autores (2018)

As informações referentes aos parâmetros de soldagem, para o estudo do fator de operação, tempo de arco aberto, tempo de soldagem e taxa de deposição do processo de soldagem GMAW com arame A5.18 ER70S-6 são apresentadas conforme Tabela 3.

Tabela 3 - Parâmetros de soldagem com arame A5.18 ER70S-6

\begin{tabular}{ll}
\hline Diâmetro do arame & $1,2 \mathrm{~mm}$ \\
Posição de soldagem & Plana \\
Gás de Proteção ativo & $75 \%$ de argônio e 25\% \\
Tipo de transferência metálica & Curto-Circuito \\
Velocidade da alimentação do arame & $6,8 \mathrm{~m} / \mathrm{min}$ \\
Tensão & $29,0 \mathrm{~V}$ \\
Corrente & $200 \mathrm{~A}$ \\
\hline
\end{tabular}

Fonte: Os Autores (2018) 


\section{(1) 1 anter}

Fotografias 2a, 2b, 2c, 2d e 2e - Processo de soldagem FCAW com arame A5.20 71T-1
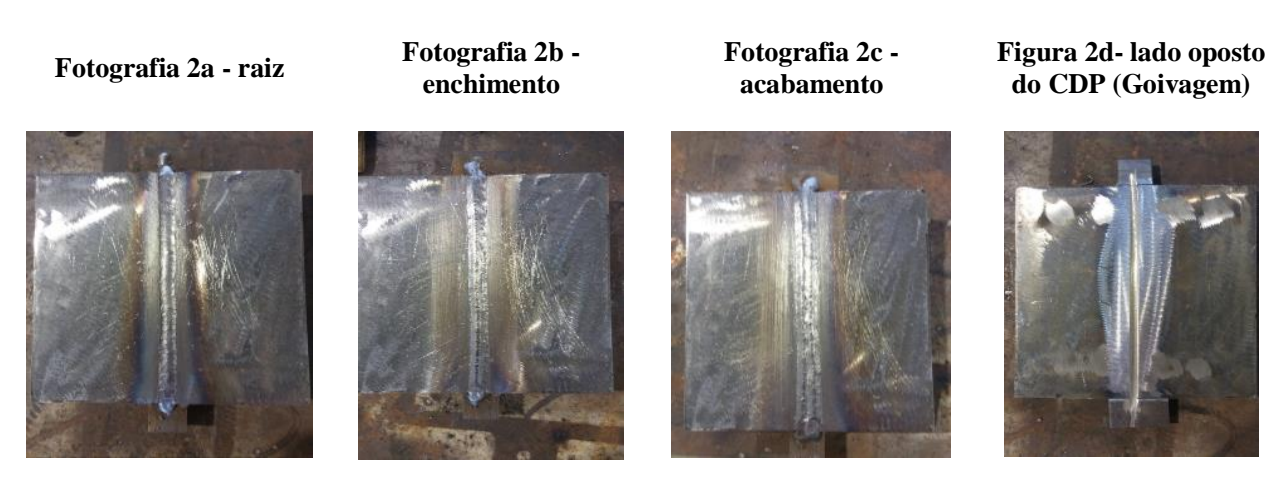

Figura 2e -

acabamento do lado oposto do CDP (Goivagem)

Fonte: Os Autores (2018)

As informações do processo de soldagem FCAW com arame A5.20 E71T-1 consideradas referente os parâmetros de soldagem, para o estudo do fator de operação, tempo de carco aberto, tempo de soldagem e taxa de deposição conforme Tabela 4.

Tabela 4 - Parâmetros de soldagem com arame A5.20 E71T-1

\begin{tabular}{ll}
\hline Diâmetro do arame & $1,2 \mathrm{~mm}$ \\
Posição de soldagem & Plana \\
Gás de Proteção ativo & $100 \% \mathrm{CO}_{2}$ \\
Tipo de transferência metálica & Curto-Circuito \\
Velocidade da alimentação do arame & $8,5 \mathrm{~m} / \mathrm{min}$ \\
Tensão & $25,0 \mathrm{~V}$ \\
Corrente & $200 \mathrm{~A}$ \\
\hline
\end{tabular}

Fonte: Os Autores (2018)

Os termos citados são variáveis que influenciam nas características do cordão de solda, dentre elas, enfatiza-se a corrente de soldagem, tensão, comprimento e diâmetro dos arames de soldagem, inclinação da tocha de soldagem, proteção gasosa, velocidade de alimentação do arame, taxa de deposição, tempo de arco aberto e tempo de soldagem. Essas são as principais variáveis dos processos de soldagem GMAW e FCAW.

Através da coleta dos dados de cada experimento laboratorial, realizou-se o estudo da taxa de deposição e de cada processo de soldagem. Assim, para cada amostra de aço ASTM A36 executou-se cinco cordões de solda com parâmetros diferentes. As fotografias 1 (1a, 1b, 1c, $1 \mathrm{~d}$ e 1e) e fotografias 2 ( $2 \mathrm{a}, 2 \mathrm{~b}, 2 \mathrm{c}, 2 \mathrm{~d}$ e $2 \mathrm{e}$ ) demonstraram os corpos de provas soldados, sendo que os três primeiros cordões foram feios em um lado da chapa e a goivagem é o último 


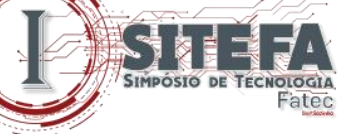

cordão do lado oposta da chapa, tal como apresentadas na sequência das respectivas fotografias.

Para realização dos cálculos da taxa de deposição foram utilizadas, conforme Brito e Paranhos (2005), as equações 1, 2, 3 e 4 apresentadas a seguir:

- $\quad$ Equação 1:

$$
F O=\frac{T A A}{T T S} \times 100
$$

$\mathrm{FO}=$ Fator de operação $(\%)$

TAA $=$ Tempo de arco aberto $(\mathrm{s})$

TTS $=$ Tempo total de soldagem $(\mathrm{s})$

- $\quad$ Equação 2:

$T A A=\frac{C S}{V S}$

TAA $=$ Tempo de arco aberto $(\min )$

$\mathrm{CS}=$ Comprimento da Solda $(\mathrm{cm})$

VS $=$ Velocidade de Soldagem $(\mathrm{cm} / \mathrm{min})$

- $\quad$ Equação 3:

$T S(h / m)=\frac{P M D}{T D \times F O}$

$\mathrm{TS}=$ Tempo de soldagem $(\mathrm{h})$

PMD = Peso do metal depositado $(\mathrm{kg})$

$\mathrm{TD}=$ Taxa de deposição $(\mathrm{kg} / \mathrm{h})$,

$\mathrm{FO}=$ Fator de operação (\%). 


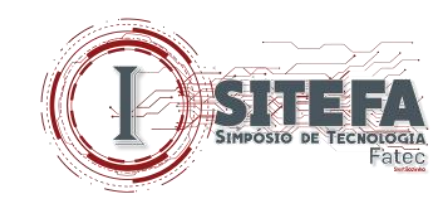

- $\quad$ Equação 4:

$T D=\frac{P F \cdot P I}{T A A}$

$\mathrm{TD}=$ Taxa de deposição $(\mathrm{kg} / \mathrm{h})$

$\mathrm{PF}=$ Peso Final $(\mathrm{kg})$

$\mathrm{PF}=$ Peso inicial $(\mathrm{kg})$

TAA $=$ Tempo de arco aberto $(\mathrm{h})$

Para execução dos experimentos laboratoriais os valores do tempo de arco aberto foram realizados através do controle, que pode ser feito através de cronometro ou calculado. Para esse estudo utilizou-se o cronometro para definir o tempo do arco aberto e o tempo de soldagem.

\section{RESULTADOS E DISCUSSÃO}

A partir dos parâmetros coletados durante a execução de cada processo, para comparação da taxa de deposição entre o arame sólido A5.18 ER70S-6 e arame tubular A5.20 E71T-1, as principais sequências de cordões de solda, técnicas operatórias, características elétricas (corrente, tensão e outras), ferramentas e equipamentos utilizados, obteve-se os dados apresentados conforme Tabelas 3 e 4 .

\subsection{Cálculo da taxa de deposição, fator de operação, tempo de arco aberto e tempo de}

\section{Soldagem}

As Tabelas 5 e 6 apresentam os cálculos exatos de cada equação, aplicados para os processos de soldagem GMAW e FCAW onde comparou-se a taxa de deposição dos arames estudados e determinou-se a quantidade de material depositado nas amostras. Para isso, as equações servem para analisar: 


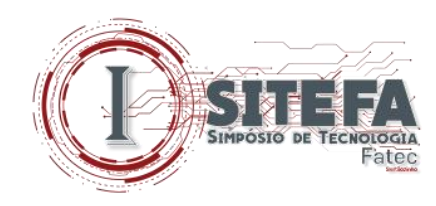

- $\quad$ O cálculo do fator de operação que é determinado pela razão do tempo em que o soldador ou operador permanece com o arco aberto e dividido pelo tempo total de soldagem, multiplicado por $100 \%$, conforme equação 1 .

- $\quad$ O tempo de arco aberto que é o tempo exato que foi empregado para depositar a solda, como mostra a equação 2 .

- $\quad$ O tempo de soldagem (h) que é o peso do material depositado (k/h) dividido pela taxa de deposição $(\mathrm{kg} / \mathrm{m})$ vezes o fator de operação $(\%)$, conforme equação 3.

A taxa de deposição que é a subtração do peso final com o peso inicial da chapa, dividido pelo tempo de arco aberto conforme equação 4.

Tabela 5 - Cálculos do processo de soldagem GMAW

\begin{tabular}{|c|c|c|c|}
\hline $\begin{array}{c}\text { Cálculo da Taxa de } \\
\text { deposição do }\end{array}$ & $\begin{array}{c}\text { Cálculo do fator de } \\
\text { operação }\end{array}$ & $\begin{array}{c}\text { Cálculo do tempo de arco } \\
\text { aberto }\end{array}$ & $\begin{array}{c}\begin{array}{c}\text { Cálculo do tempo de } \\
\text { soldagem }\end{array} \\
\end{array}$ \\
\hline $\mathrm{TD}(\mathrm{kg} / \mathrm{h})=\frac{\mathrm{PF}-\mathrm{PI}}{\mathrm{TAA}}$ & $\mathrm{FO}(\%)=\frac{\mathrm{TAA}}{\mathrm{TTS}} \times 100$ & $\mathrm{TAA}(\min )=\frac{\mathrm{CS}}{\mathrm{VS}}$ & $T S(h / m)=\frac{P M D}{T D \times F O}$ \\
\hline $\mathrm{TD}=\frac{5,166-5,031}{0,103}$ & $\mathrm{FO}=\frac{6,17}{45} \times 100$ & $\mathrm{TAA}=\frac{250}{40,5}$ & $\mathrm{TS}=\underline{0,135}$ \\
\hline $\mathrm{TD}=1,31 \mathrm{~kg} / \mathrm{h}$ & $\mathrm{FO}=13,71 \%$ & $\mathrm{TAA}=6,17 \mathrm{~min}$. & $\mathrm{TS}=0,75 \mathrm{~h} \equiv 45 \mathrm{~min}$ \\
\hline
\end{tabular}

Fonte: Os Autores (2018)

Tabela 6 - Cálculos do processo de soldagem FCAW

\begin{tabular}{|c|c|c|c|}
\hline $\begin{array}{c}\text { Cálculo da taxa de } \\
\text { deposição }\end{array}$ & $\begin{array}{c}\text { Cálculo do fator de } \\
\text { soldagem }\end{array}$ & $\begin{array}{c}\text { Cálculo do tempo de arco } \\
\text { aberto }\end{array}$ & $\begin{array}{c}\begin{array}{c}\text { Cálculo do tempo de } \\
\text { soldagem }\end{array} \\
\end{array}$ \\
\hline $\mathrm{TD}(\mathrm{kg} / \mathrm{h})=\frac{\mathrm{PF}-\mathrm{PI}}{\mathrm{TAA}}$ & $\mathrm{FO}(\%)=\frac{\mathrm{TAA}}{\mathrm{TTS}} \times 100$ & $\mathrm{TAA}(\min )=\frac{\mathrm{CS}}{\mathrm{VS}}$ & $T S(h / m)=\frac{P M D}{T D \times F O}$ \\
\hline $\mathrm{TD}=\frac{5,146-5,031}{0,093}$ & $\mathrm{FO}=\frac{5,58}{35} \times 100$ & $\mathrm{TAA}=\frac{250}{44,8}$ & $\mathrm{TS}=\frac{0,115}{\left.1,24 \times \frac{(15,94}{100}\right)}$ \\
\hline $\mathrm{TD}=1,24 \mathrm{~kg} / \mathrm{h}$ & $\mathrm{FO}=15,94 \%$ & $\mathrm{TAA}=5,58 \mathrm{~min}$. & $\mathrm{TS}=0,5808 \mathrm{~h} \equiv 35 \mathrm{~min}$. \\
\hline
\end{tabular}

Fonte: Os Autores (2018)

A partir dos cálculos realizados para comparação da taxa de deposição entre o arame sólido e tubular onde o resultado da taxa de deposição se expressa por $\mathrm{kg} / \mathrm{h}$ de material depositado. A utilização do diâmetro dos arames, posição de soldagem, corrente e o tipo de 


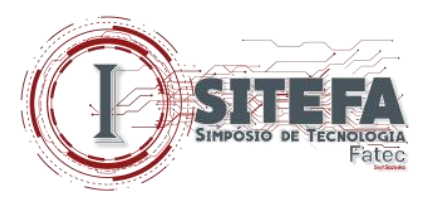

transferência foram a mesma para ambos os arames (sólido e tubular), já os parâmetros como a velocidade da alimentação do arame, tensão, tipo de gás, foram diferentes para os arames (sólido e tubular). Os gráficos a seguir representam uma comparação da taxa de deposição. (Gráfico 1) taxa de deposição, (gráfico 2) fator de soldagem, (gráfico 3) tempo de arco aberto, (gráfico 4) tempo de soldagem.

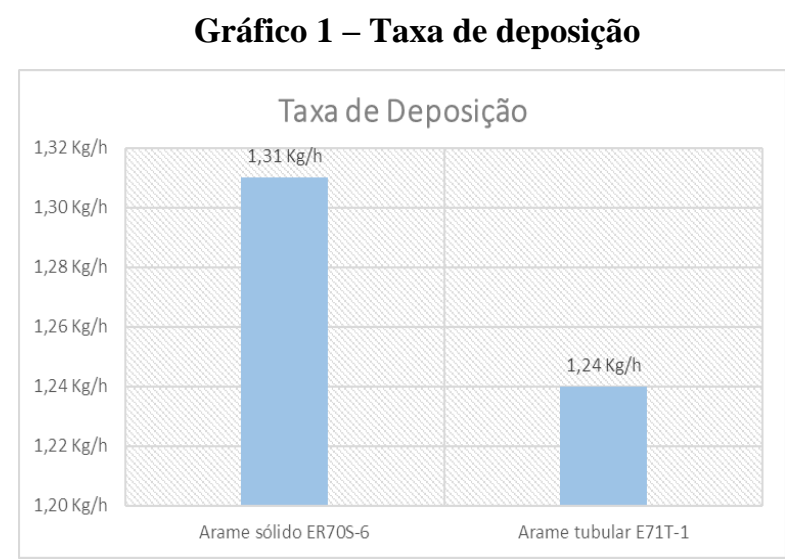

Fonte: Os Autores (2018)

Gráfico 3 - Tempo de arco aberto

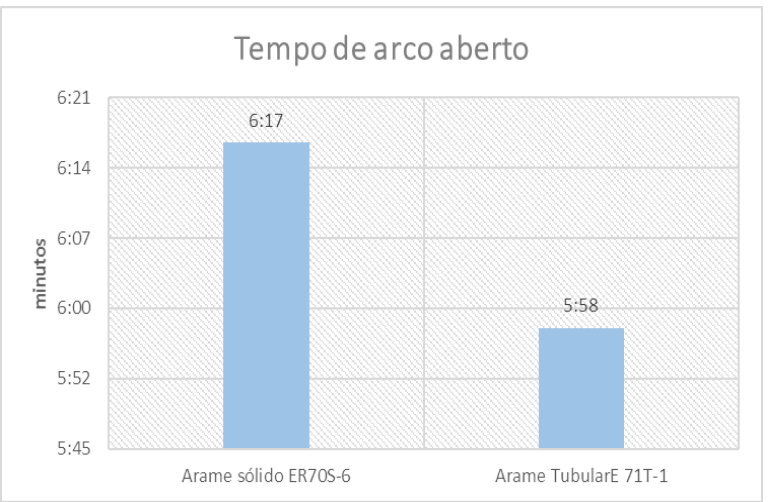

Fonte: Os Autores (2018)
Gráfico 2 - Fator de soldagem

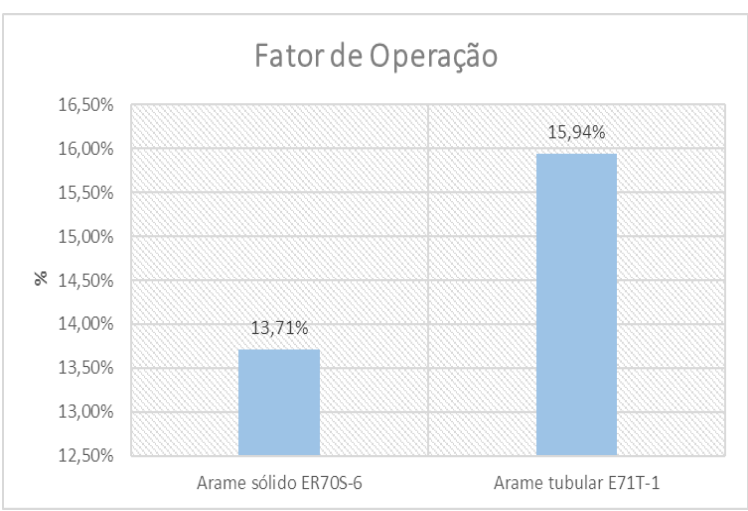

Fonte: Os Autores (2018)

Gráfico 4 - Tempo de soldagem

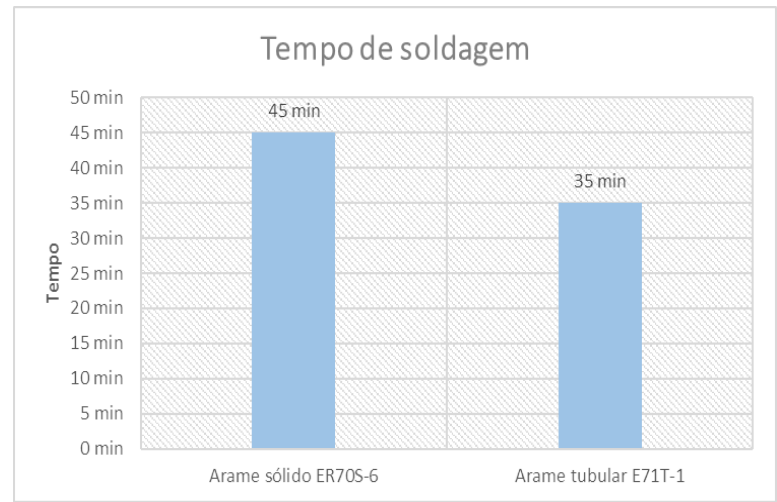

Fonte: Os Autores (2018)

Analisando-se os gráficos onde estão apresentados os valores calculados da taxa de deposição (gráfico1), fator de operação (gráfico 2), tempo de arco aberto (gráfico 3) e tempo de soldagem (gráfico 4), pode-se apresentar os seguintes resultados: 


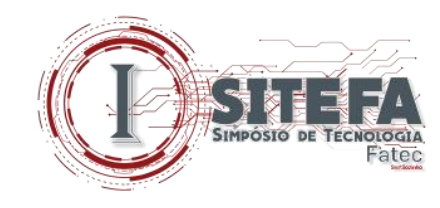

a) ao analisar os dados da taxa de deposição, identificou-se que a taxa de deposição do arame sólido ER70S-6 apresentou uma variação percentual de 5,34\% na taxa de deposição perante o arame tubular E71T-1, um valor bem significativo para o processo;

b) já o gráfico 2 representa o fator de operação onde obteve-se uma variação percentual de 13,99\% no desempenho do arame tubular E71T-1 apresentando um melhor aproveitamento que o arame ER70S-6;

c) no gráfico 3 apresenta-se o tempo de arco aberto, onde o arame E71T-1 obteve um melhor desempenho do tempo de arco aberto, gasto para deposição da solda, que o arame ER70S-6.

d) na análise do gráfico 4 é possível verificar que o tempo de soldagem do arame tubular ficou em 0:35 minutos, apresentando um melhor tempo em relação ao arame sólido com 0:45 minutos de tempo de soldagem, apresentando uma variação percentual de tempo de $28,57 \%$.

\section{CONCLUSÃO}

Este artigo apresentou os resultados da pesquisa cujo objetivo é comparar o processo de soldagem $G M A W$ e $F C A W$ com os arames maciço e tubular, em função da taxa de deposição.

A partir das análises dos materiais, métodos e cálculos realizados foi possível concluir que a taxa de deposição foi superior para o arame ER70S-6 em relação ao arame E71T-1 na faixa de regulagem de transferência por curto-circuito e tensões de 29,0 V do arame sólido e 25,0 V do arame tubular e nas condições da corrente e velocidade de alimentação dos arames utilizados.

A pesquisa também conclui que o fator de operação do arame tubular apresentou um melhor desempenho em função do operador manter um maior tempo do arco aberto soldando obtendo uma variação percentual de 14\% em vantagem ao arame sólido, propiciando melhor rendimento e maior produtividade. Outra variável destacada é a velocidade de alimentação que teve ação direta no rendimento do processo e na atuação do arco com menor intensidade de tensão. Busca de um aumento na produtividade e consequentemente na redução do tempo de soldagem. 


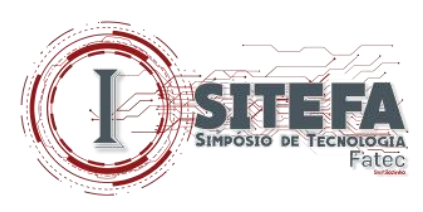

\section{REFERÊNCIAS}

ASSOCIAÇÃO BRASILEIRA DE NORMAS TÉCNICAS (ABNT). NBR 13043: Soldagemnúmeros e nomes de processo. Rio de Janeiro: ABNT, 1993.

BARROS, Aidil Jesus Paes de; LEHFELD, Neide Aparecida de Souza. Fundamentos de metodologia: um guia para iniciação cientifica. São Paulo: McGraw-Hill, 1986.

BRACARENSE, Alexandre; MODENESI, Paulo; MARQUES, Paulo José. Soldagem: fundamentos e Tecnologia. 3. ed. Belo Horizonte: Editora UFMG, 2009.

BRITO, José de Deus; PARANHOS, Ronaldo. Como determinar os custos da soldagem. Rio de Janeiro: Paranhos, 2005.

COLPAERT, H. . Metalografia dos Produtos Siderúrgicos Comuns. 4. ed. São Paulo: Blucher, 2008.

GOMES, E. B. Análise do comportamento da soldagem por curto circuito aplicado ao processo eletrodo tubular através da metodologia Taguchi. 2006. 119 p. Dissertação (Mestrado em Engenharia Mecânica) - Departamento de Engenharia Mecânica, Universidade Federal de Itajubá, Itajubá. 2006.

GERDAU. Certificado de Qualidade/ Mill Test/ Quality Certificate. 2017.

LINCOLN ELETRIC BRASIL. Composição química dos arames maciço ER70S-6 e arame tubular E71T-1. Disponível em: <http://www.lincolnelectric.com.br/upload/lw71(2).pdf> e <http://www.lincolnelectric.com.br/upload/merit-s-6(1).pdf>. Acesso em: 11 ago. 2018.

PRODANOV, Cleber Cristiano; FREITAS, Ernani Cesar. Metodologia do Trabalho Científico: métodos e técnicas da pesquisa e do trabalho acadêmico. 2. ed. Novo Hamburgo: Feevale, 2013.

RODRIGUES, L. O., PAIVA, A. P.; COSTA, S. C. Otimização do processo de soldagem com eletrodo tubular através da análise da geometria do cordão de solda. In: Soldagem \& Inspeção, v. 13, n. 2, p. 118-127, 2008

WAINER, Emílio; BRANDI, Sérgio Duarte; MELLO, Fábio Décourt Homem de. Soldagem: processo e metalurgia. São Paulo: Edgard Blucher, 2015.

Soldagem: processo e metalurgia. São Paulo: Edgard Blucher, 2004. 